\title{
Adaptive control design for a Mimo chemical reactor
}

\author{
Yasabie Abatneh, Omprakash Sahu* \\ Department of Chemical Engineering, KIOT, Wollo University, Kombolcha, Ethiopia
}

Email address:

ops0121@gmail.com(O.Sahu)

\section{To cite this article:}

Yasabie Abatneh, Omprakash Sahu. Adaptive Control Design for a Mimo Chemical Reactor. Automation Control and Intelligent Systems. Vol. 1, No. 3, 2013, pp. 64-70. doi: 10.11648/j.acis.20130103.15

\begin{abstract}
The major disadvantage of non-adaptive control systems is that these control systems cannot cope with fluctuation in the parameters of the process. One solution to this problem is to use high levels of feedback gain to decrease the sensitivity of the control system. However high gain controllers have two major problems: large signal magnitude and closed loop instability. The solution to this problem is to develop a control system that adapts to changes in the process. This paper presents the design of adaptive controller to a Multi Input Multi Output (MIMO) chemical reactor. The proposed adaptive controller is tested by using Math lab Simulink program and its performance is compared to a conventional controller for a different situation. The paper demonstrated that while the adaptive controller exhibits superior performance in the presence of noise the convergence time is typically large and there is a large overshoot. The results from the case study indicate that the use of adaptive controller can be extended to process with inverse response. For such process the adaptive controller will be superior to the conventional controller even without parameters change in the process. Although the conventional controller has the smaller response time, it is incapable of eliminating the inverse response.
\end{abstract}

Keywords: Process, Controller, Data, Model

\section{Introduction}

In common sense, 'to adapt' means to change a behavior to conform to new circumstances. Intuitively, an adaptive controller is thus a controller that can modify its behavior in response to the change in dynamics of the process and the character of the disturbances [1]. Adaptive control systems have been in existence for over thirty years, and a wide range of approaches have been developed [2]. The core element of all the approaches is that they have the ability to adapt the controller to accommodate changes in the process. This permits the controller to maintain a required level of performance in spite of any noise or fluctuation in the process. There are wide ranges of adaptive control methods currently in use but the objectives are the same that to provide an accurate representation of the process at all times [3]. An adaptive system has maximum application when the plant undergoes transitions or exhibits non-linear behavior and when the structure of the plant is not known. Gain scheduling is one form of adaptive control but it requires knowledge about all the process to be effective. Another alternative is to adapt the controller's parameters or when a model is available to use the model identification error to tune the controller's parameters [4]. Consequently, tuning of the controller is indirect and necessarily requires an accurate model of the process for satisfactory performance. Adaptive control systems are currently used in many operations and one of these operations is chemical process. There are two main reasons why adaptive controller is nee chemical processes. First, most chemical processes are non linear. Therefore, the linear zed models that are used to design linear controllers depend on the particular steady state (around which the process is linearized) .It is clear that as the desired steady state operation of a process changes, the 'best' values of the controller's parameters change. This implies the need for controller adaptation. Second, most of the chemical processes are non stationary (i.e. their characteristics change with time). Typical examples are the decay of the catalyst activity in a reactor and the decrease of the overall heat transfer coefficient in a heat exchanger due to fouling. These change leads again to deterioration in the performance of linear controller which was designed using some nominal values for the process parameters, thus requiring adaptation of the controller parameters. The purpose of this paper is to design and simulate a Model Reference Adaptive control (MRAC) for a multiple inputs 
multiple outputs chemical reactor [5]. The paper includes the following parts: section 2 provides an overview of model reference adaptive controller and design and simulation adaptive controller and comparison of performance of adaptive controller random-adaptive (conventional) controller. In the last section a case study which considered the control of Van de Vausee reactor will be demonstrated.

\section{Adaptive Control Design and Simulation}

This section provide three sets: the first covers the design of model reference adaptive control and its implementation in simulink. The second and the third compare the performance of an adaptive control to a conventional controller without noise and with noise respectively.

\subsection{Basic Adaptive Controller}

This is to provide the implementation of a basic adaptive controller using simulink. The first item that must be defined is the plant that is to be controlled. A CSTR with a general reaction $\mathrm{A} \rightarrow \mathrm{B}$ is the system to be controlled and the control variables are concentration of $\mathrm{A}$ and temperature of the reactor.

The transfer function for the two SISO systems (concentration and temperature of reaction control) are obtained as

\subsubsection{Concentration Control}

$G m(s)=\frac{1.44 \mathrm{~s}^{5}+5.78 \mathrm{~s}^{4}+9 \mathrm{~s}^{3}+6.88 \mathrm{~s}^{2}+2.53 \mathrm{~s}+0.31}{\mathrm{~S}^{6}+4.41 \mathrm{~s}^{5}+7.94 \mathrm{~s}^{4}+8.42 \mathrm{~s}^{3}+4.1 \mathrm{~s}^{2}+1.19 \mathrm{~s}+0.14}$

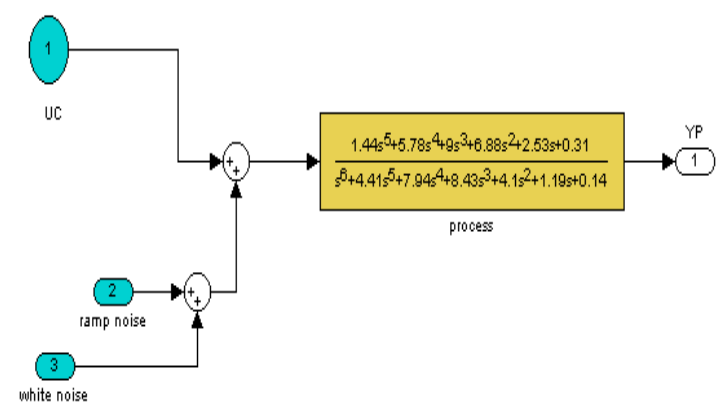

Fig 1. Simulink plant implementation

The next step is to define the model that the plant must be matched to. To determine this model we must first define the characteristics that we want the system to have. Firstly we will arbitrary select the model to be a second order model of the form:

$$
G m \quad(s)=\frac{\omega_{n}{ }^{2}}{s^{2}+2 \omega_{n} \xi s+\omega_{n}{ }^{2}}
$$

We must then determine the damping ratio $\xi$ and the natural frequency $\omega$ n to give the required performance characteristics. For the concentration control a maximum overshoot (Mp) of 5\% and a settling time (Ts) of less than 2 seconds are selected. We can use the equation below to determine the required damping ratio and natural frequency of the system.

$$
\begin{gathered}
\xi=\frac{\ln \mathrm{p}_{\mathrm{p}} / 100}{-\pi} \sqrt{\frac{1}{1+\left[\frac{\ln \mathrm{p}_{\mathrm{p}} / 100}{-\pi}\right]^{2}}} \\
\omega_{n}=\frac{3}{T_{s} \xi}
\end{gathered}
$$

Based upon these formulae we get $\xi=0.68$ and $\omega n=2.1986 \mathrm{rad} / \mathrm{s}$. The transfer function for the model is therefore

$$
G m(s)=\frac{4.834}{s^{2}+3 s+4.834}
$$

Note that we have defined the plant we need to develop a standard controller to compare with the adaptive controller. The simulink diagram for this controller (and the plant) is shown in figure 2.

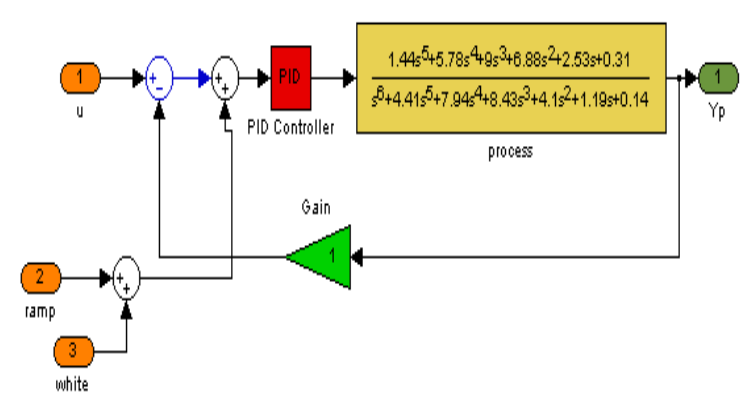

Fig 2. Simulink conventional controller

The final steps are then to implement the controller, the adaptation law and the link between the systems. 


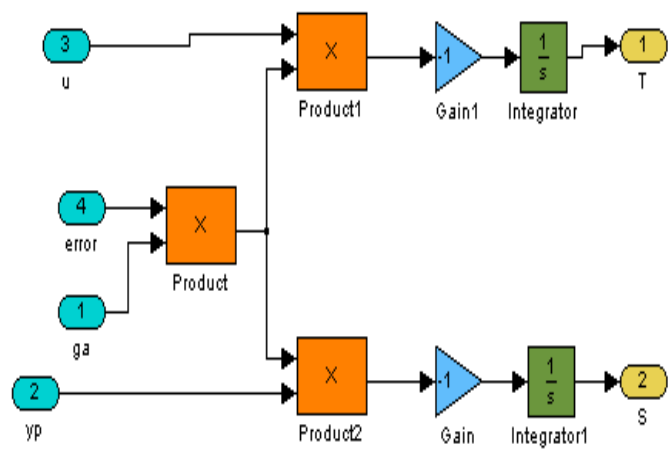

Fig 3. Simulink implementation of Lyapunov Adaptation Law

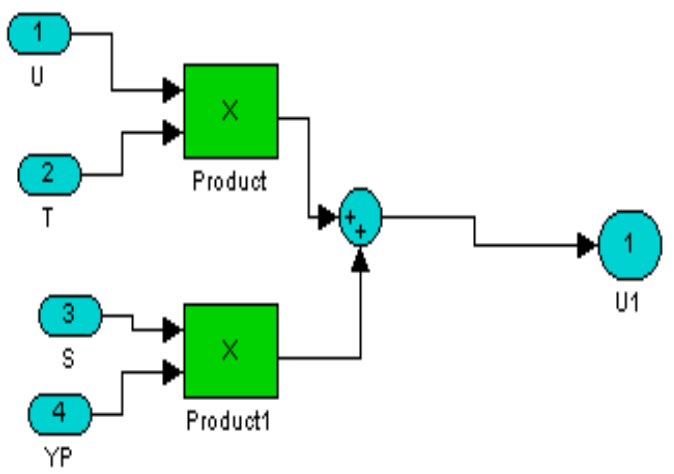

Fig 4. Simulink implementation of Controller

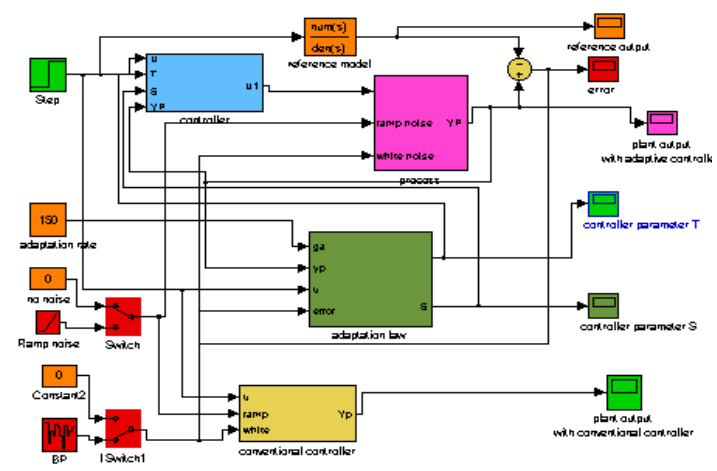

Fig 5. Simulink implementation of a Model Reference Adaptive Controller b) Temperature control

The simplified transfer function model of the process is obtained as:

$$
G_{p} 2(s)=\frac{0.3 s^{5}+1.18 s^{4}+1.96 s^{3}+1.54 s^{2}+0.62 s+0.95}{s^{6}+4.41 s^{5}+7.94 s^{4}+8.42 s^{3}+4.1 s^{2}+1.19 s+0.14}
$$

A reference model (second order) with a maximum overshot (Mp) of $2.5 \%$ and settling time (Ts) of 1 second is chosen. And the transfer function for the model is:

$$
G m(s)=\frac{15.54}{s^{2}+6 s+15.54}
$$

The implementation of the complete model reference adaptive control is identical to the previous case except $\mathrm{Gp} 1$ and $\mathrm{Gm} 1$ are replaced by $\mathrm{Gp} 2$ and $\mathrm{Gm} 2$ respectively.

The completed model permits the noise to be disabled, ramp function only, white noise only or a combination of ramp and white noise. The following parameters are plotted on graph: plant output with adaptive and with conventional control, model output, error between plant and model outputs and the controller parameters.

\subsection{ComparisonWithout Noise}

Note that the model is complete; the first task we must perform is, to compare the performance of the two controllers for a step input and no noise

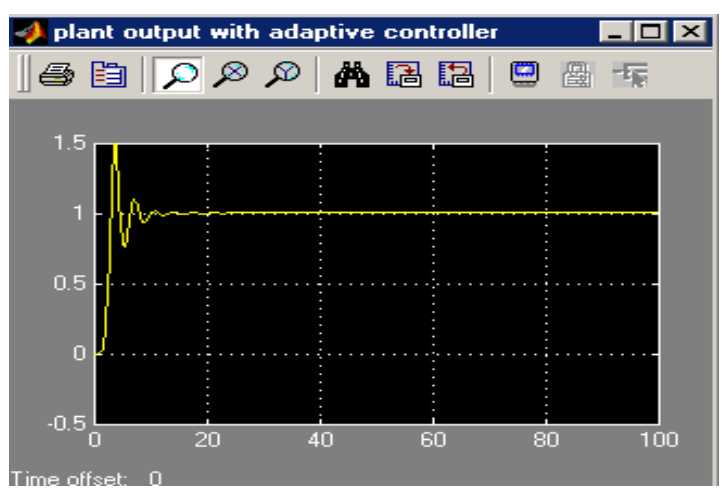

Figure 6. Plant output (concentration) with adaptive control (step input, no noise, Gamma $=0.99$ )

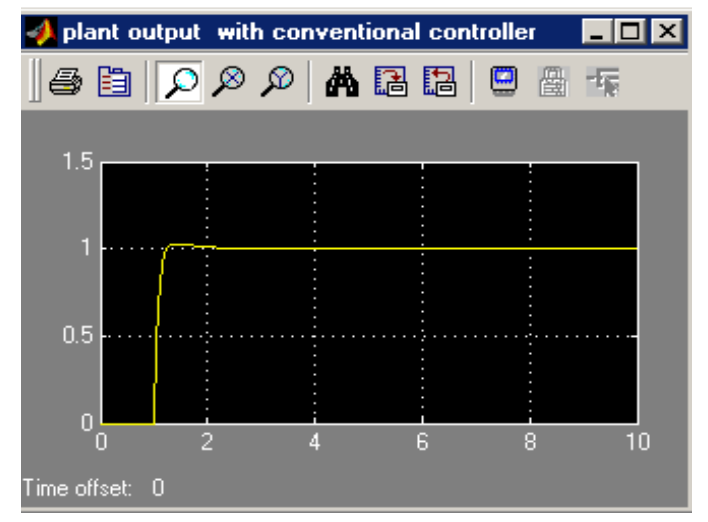

Figure 7. Plant output with conventional control (step input, no noise, Gamma $=0.99$ )

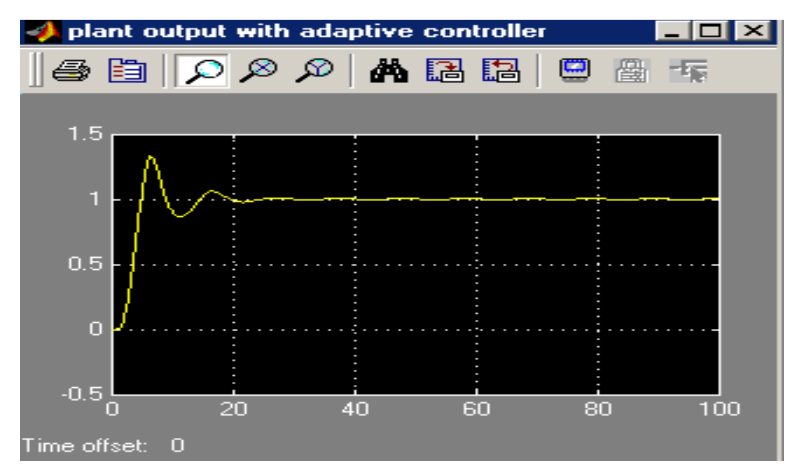

Figure 8. Plant output (temperature) with adaptive control (step input, no noise, Gamma=0.99) 


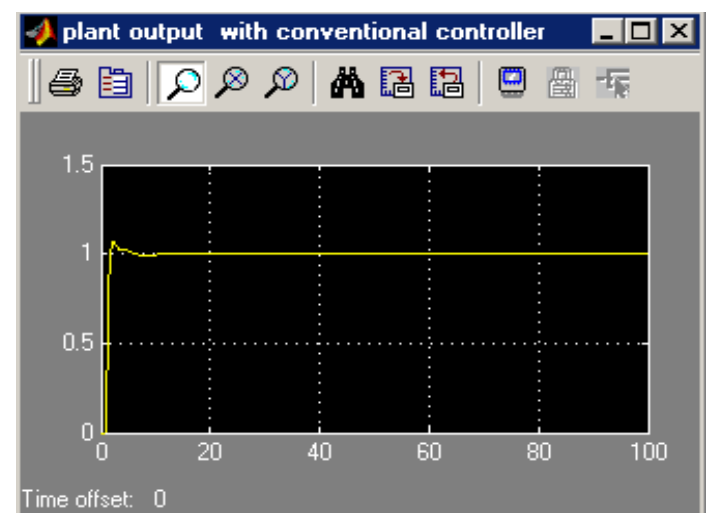

Figure 9. Plant output temperature) with conventional control (step input, no noise, Gamma $=0.99$

Looking at these graphs one of the major disadvantage of adaptive control is immediately apparent. It takes the adaptive controller nearly 20 seconds to match perfectly the output of the reference model. However the conventional controller is matched within 2 seconds. The overshoot of the adaptive controller is also excessive (of the order of $50 \%$ ) while the conventional controller has an overshoot of below $3 \%$.

One method of addressing this problem is to increase the adaptation gain (Gamma). For example, increasing the adaptation gain to 100 gives the response shown in figures 10 and 11.

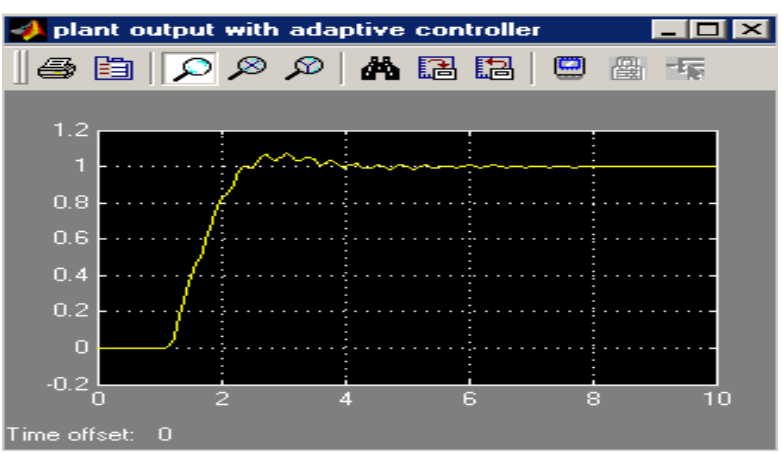

Figure 10. Plant output with adaptive control (step input, no noise, Gamma=100)

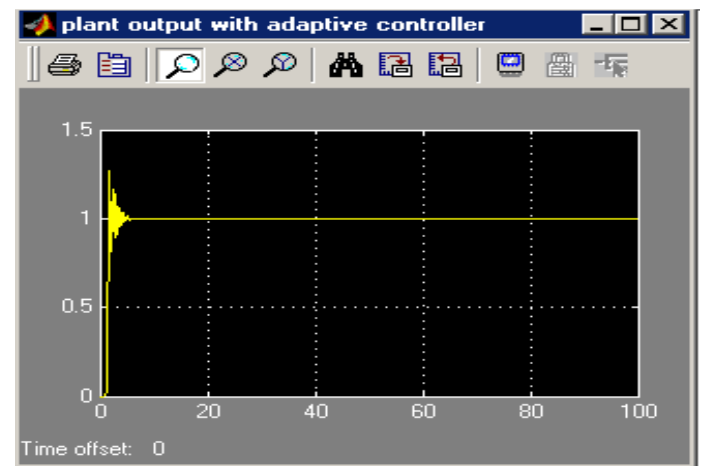

Figure 11. Plant output with adaptive control (step input, no noise, Gamma $=100$ )

This has improved the overshoot to below $10 \%$ and the settling time is now less than 10 seconds. While not perfect this is a significant improvement. Further increase in the adaptation gain does not result in an improvement of the system.

\subsection{Comparison with Noise (Comparison with Ramp Noise)}

The next logical step is to compare the performance of the two controllers in the presence of noise in the form of ramp signal, $($ slope $=1)$. The adaptation gain has been restored to 0.99 .

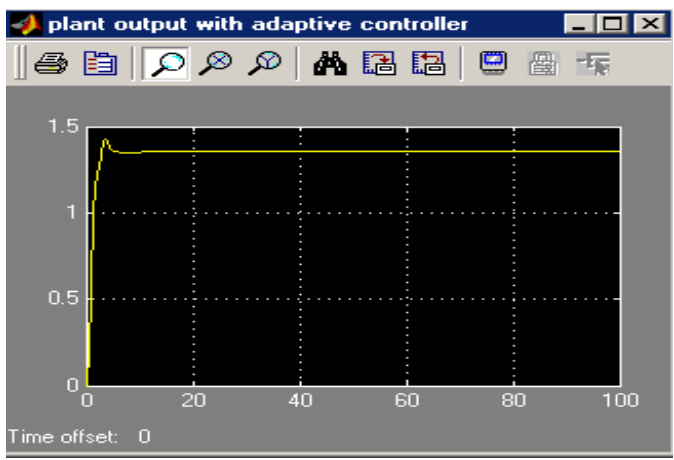

Figure 12. Plant output (concentration) with adaptive control (step input, ramp noise, Gamma $=0.99$ )

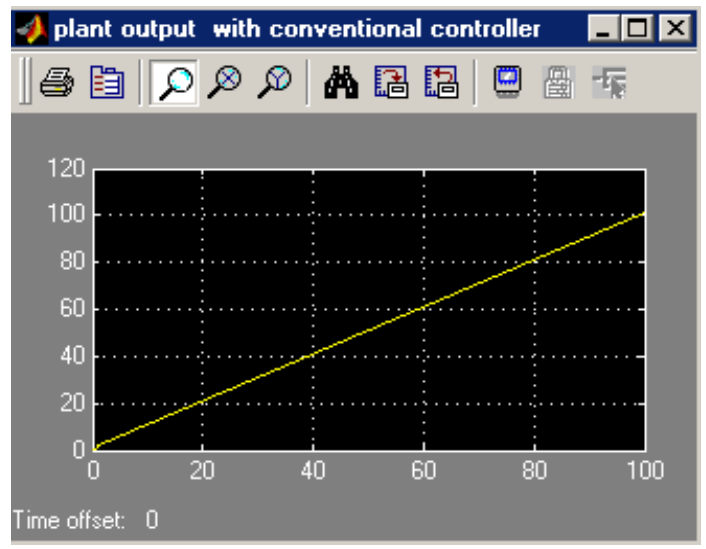

Figure 13. Plant output (concentration) with conventional control (step input, ramp noise, Gamma=0.99)

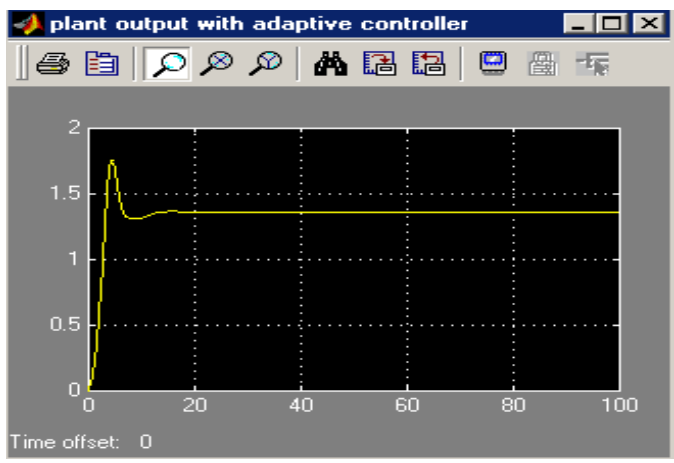

Figure 14. Plant output (temperature) with adaptive control (step input, ramp noise, Gamma $=0.99$ ) 


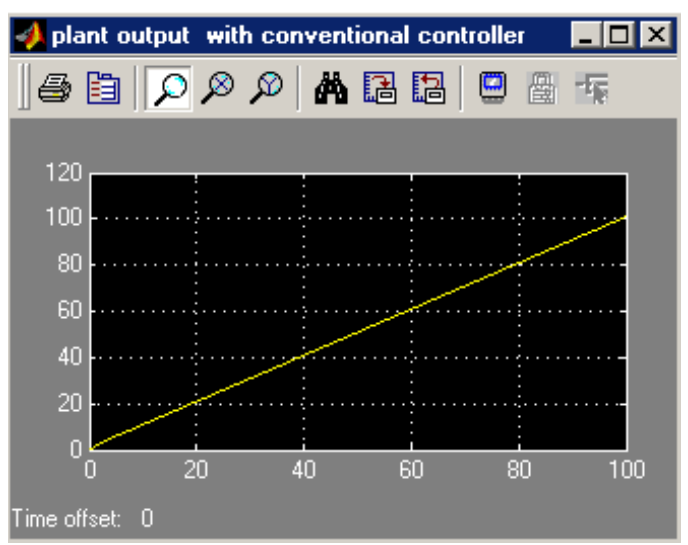

Figure 15.Plant output (temperature) with conventional control (step input, ramp noise, Gamma=0.99)

The situation begins to show the actual advantages of adaptive control. In this case the conventional controller is incapable of maintaining even a stable system. On the other hand the adaptive control manages to maintain stability. However large overshoot and offset and long settling time are present. There is also a large steady state error. As before increasing the adaptation gain to 100 reduces the overshoot to below $10 \%$, the settling time to below 5 seconds and the steady state error to zero. This is shown below in figure.

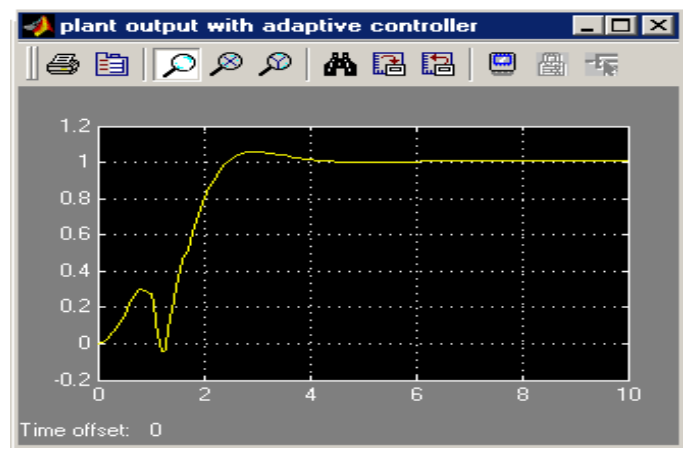

Figure 16. Plant output (concentration) with adaptive control (step input, ramp noise, Gamma $=100$ )

\subsubsection{Case Study}

Up to now, we have taken a general reaction $\mathrm{A} \rightarrow \mathrm{B}$. So it is essential to consider a specific reaction to demonstrate the proposed adaptive control scheme to a great existent.

This case study considers the control of the Van de Vusse reactor, the reaction scheme consisting of the following reactions:

$\mathrm{A} \rightarrow \mathrm{B} \rightarrow \mathrm{C}$

$2 \mathrm{~A} \rightarrow \mathrm{D}$

$\mathrm{A}=$ yclopentaddiene, $\mathrm{B}=$ Cyclopentenol

$\mathrm{C}=$ Cyclopentanediol,

$\mathrm{D}=$ dicyclopentandiene

The actual process dynamics are described by

$$
d x_{1} / d t=u\left(C_{A f}-C_{A S}-x_{1}\right)-\left(u_{s}+k_{1}+2 k_{3} C_{A s}\right) x_{1}-k_{3} x_{1}^{2}
$$

$$
d x_{2} / d t=-u\left(x_{2}+C_{B S}\right)+k_{1} x_{1}-\left(u_{s}+k_{2}\right) x_{2}
$$

Where, $\mathrm{C}_{\mathrm{AS}}$ and $\mathrm{C}_{\mathrm{BS}}$ denote the effluent concentration of component $\mathrm{A}$ and $\mathrm{B}$ at steady state, respectively. The state variables $\mathrm{x}_{1}$ and $\mathrm{x}_{2}$ are deviation variables defined by $\mathrm{x}_{1}=\mathrm{C}_{\mathrm{A}}-\mathrm{C}_{\mathrm{AS}}$ and $\mathrm{x}_{2}=\mathrm{C}_{\mathrm{B}}-\mathrm{C}_{\mathrm{BS}}$; $\mathrm{u}$ is the manipulated variable given by $u=F / V-u_{s}$, where $u s=F s / V$. the concentration of $\mathrm{A}$ in the feed stream, denoted by $\mathrm{C}_{\mathrm{Af}}$ and is equal to $10 \mathrm{~mol} / \mathrm{L}$. The reactor volume, $\mathrm{V}$, is $7 \mathrm{~L}$ and the rate constants are $\mathrm{k}_{1}=0.8333 \mathrm{~min}-\mathrm{s}, \mathrm{k}_{2}=1.6667 \mathrm{~min}-\mathrm{s}$ and $\mathrm{k}_{3}=0.1667$ L.mol-1.min-1. It is known that the process is at steady state with $\mathrm{F}_{\mathrm{s}}=4 \mathrm{~L} / \mathrm{min}, \mathrm{C}_{\mathrm{AS}}=3 \mathrm{~mol} / \mathrm{L}$ and $\mathrm{C}_{\mathrm{BS}}=1.117$ $\mathrm{mol} / \mathrm{L}$ initially. The control objective is to regulate the concentration of $\mathrm{B}, \mathrm{x}_{2}$, by manipulating the dilution rate, Here; it is assumed that the original nonlinear characteristics are unknown and only the process nominal transfer function

$$
G_{p}(s)=\frac{0.5848(-0.3549 s+1)}{0.1858 s^{2}+0.8627 s+1}
$$

Is known and is available for design. The response for step change in the input is shown in figure 17

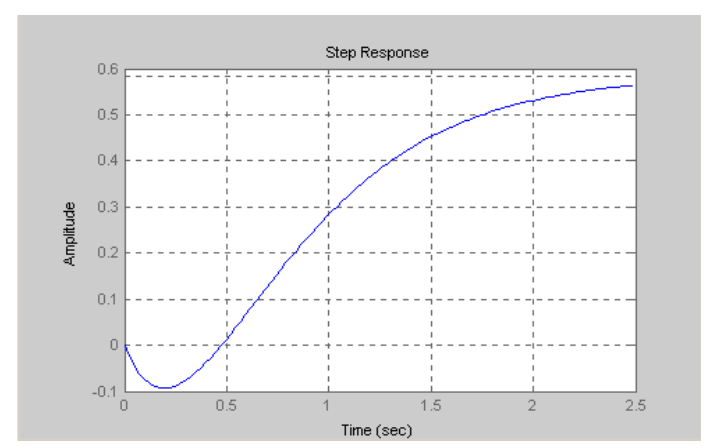

Figure 17. Response for a step change in the input

For the adaptive control system, a reference model (second order) with maximum overshoot $(5 \%)$ and settling time of 2 second is chosen and the transfer function for the model is:

$$
G_{P}(s)=\frac{4.834}{s^{2}+3 s+4.834}
$$

Controller setting for the non-adaptive controller is done using Ziegler-Nicholas technique and the best controller parameters are found to be $\mathrm{Kc}=3, \tau \mathrm{I}=1$ and $\tau \mathrm{d}=0.167$.

Using the above information the performances of the two controllers are compared for different situation.

\subsection{Comparison without Noise}

First, the performances of the two controllers without noise are compared. The responses for a step change (0.1) in the input are shown in figure 18. and figure 19. 


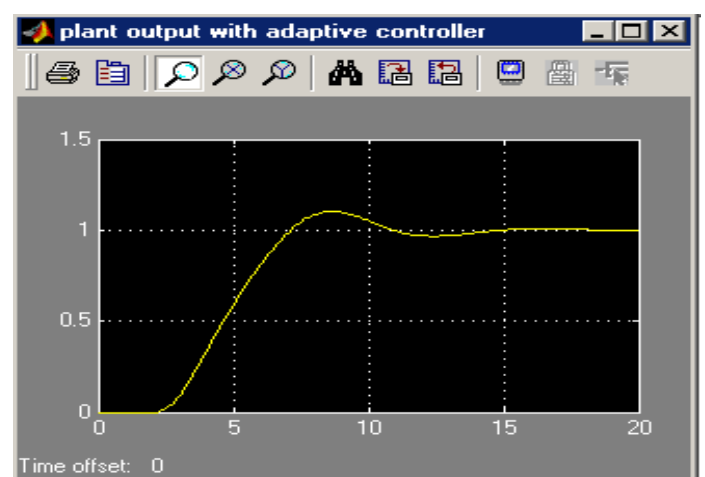

Figure 18. Plant output with adaptive controller (step input, no noise, $\gamma=0.5$ )

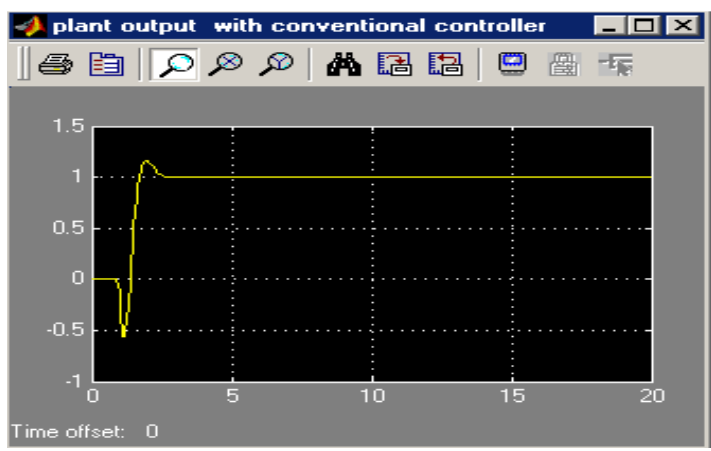

Figure 19. Plant output with conventional controller (step input, no noise)

Looking at these two graphs, the advantage of adaptive controller can be seen as it eliminates the inverse response. Although the conventional controller has the smaller response time (2 second) compared to the adaptive controller ( 7 second), it is incapable of eliminating the inverse response. The response time for the adaptive controller can be decreased at a cost of initial oscillation. Figure 20 shows the effect of increasing the adaptation rate to 0.75 .

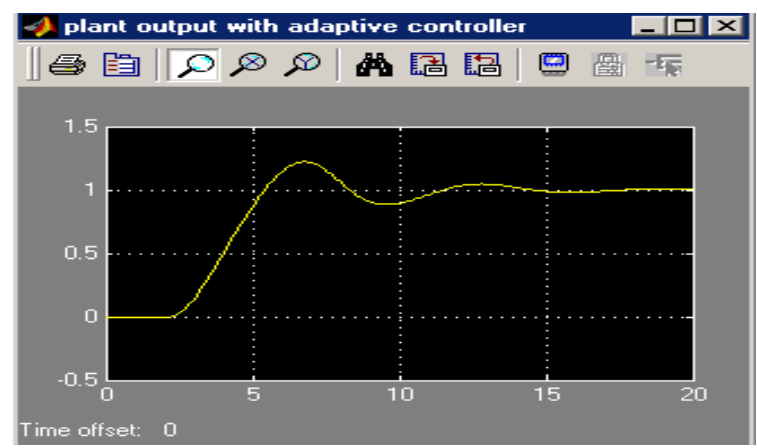

Figure 20. Plant output with adaptive controller (step input, no noise, $\gamma=0.75$ )

\subsubsection{Comparison with Ramp Noise}

The next task to be done is to compare the performances when there is a disturbances or a change in the process parameters. The responses for a step change in the input with a ramp noise $($ slope $=0.1)$ are shown in the next two figures.

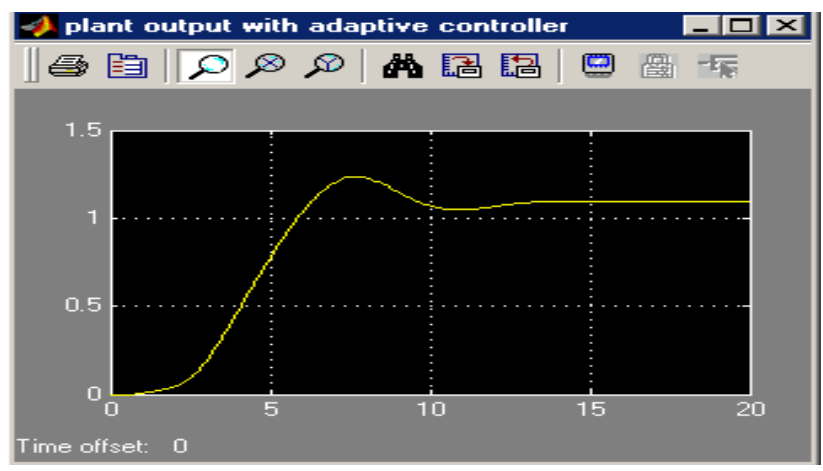

Figure 21. Plant output with adaptive controller (step input, ramp noise, $\gamma=0.5)$

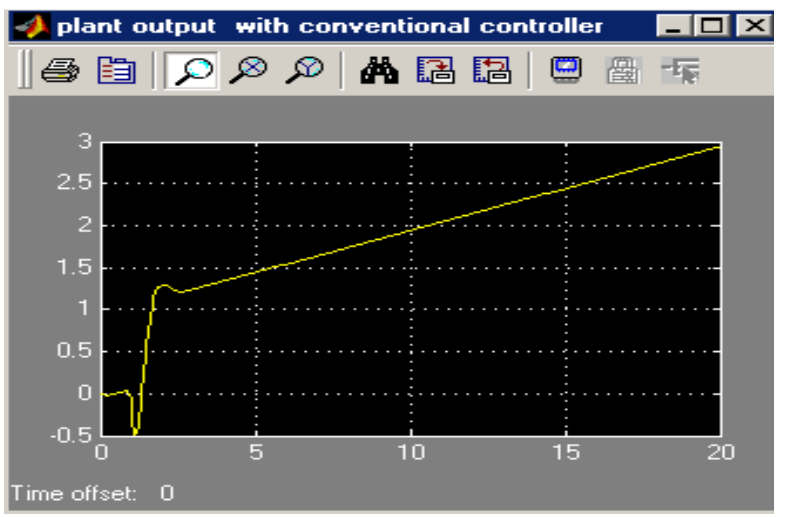

Figure 22. Plant output with conventional controller (step input, ramp noise)

From the above two figures it can be seen that the conventional controller is incapable of maintain even stability. Although there is a large steady state error, the adaptive controller gives a better plant response. But the steady state error can be eliminated by increasing the adaptation rate. This is shown in the next figure.

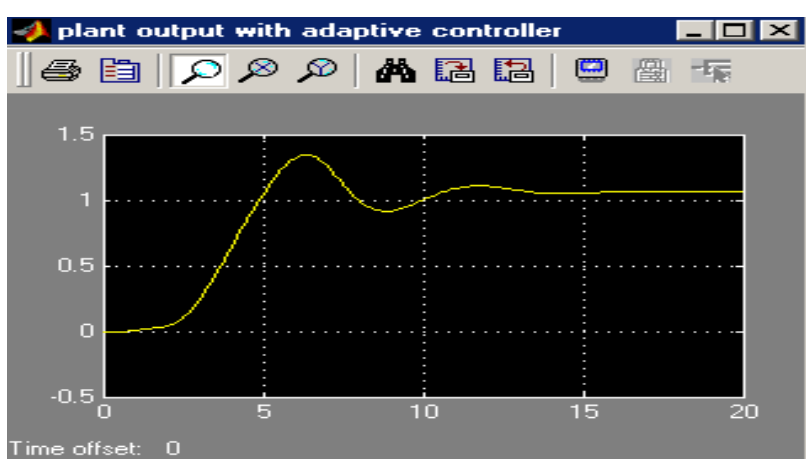

Figure 23. Plant output with adaptive controller (step input, ramp noise, $\gamma=0.75$ )

\section{Conclusion}

The use of multiple model and adaptive controller are attractive especially when the process is known to transition to unknown operating states. It is also intuitive that a fixed parameter controller (conventional controller) may not provide satisfactory closed loop control. The non adaptive 
model can provide speed whenever its parameters are close to those of the process while the adaptive model can provide accuracy because its parameters are permitted to adapt.

The paper has aimed to provide an understanding of how to implement an adaptive controller and to compare an adaptive controller with a conventionally designed controller in various situations. The paper demonstrated that while the adaptive controller exhibits superior performance in the presence of noise the convergence time is typically large (greater than 10 seconds) and there is large overshoot. These two problems are due to the adaptive controller failing to adapt fast enough to force the plant to match the model. Increasing the adaptation rate improves the performance of the adaptive controller at the cost of increased oscillation. One interesting observation is that the presence of noise increase the time required for the adaptive controller to converge. The probable reason for this is that the presence of the noise provides the adaptive controller with more of signals to process.

The results from the case study indicate that the use of adaptive controller can be extended to process with inverse response. For such process the adaptive controller will be superior to the conventional controller even without parameters change in the process. Although the conventional controller has the smaller response time, it is incapable of eliminating the inverse response.

\section{References}

[1] B.Wayne Bequette, process dynamic modelling, analysis and simulation; Precentice Hall:NJ,1998

[2] G. Stephanopolus, Chemical process control; prentice Hall of India private limited, New Delhi, 2002.

[3] William L.Luyben Process Modeling, Simulation and Control for Chemical Engineer;MCGraw-Hill,Inc., 1990

[4] C.T. Chen; Direct Adaptive Control of Chemical Process System; Industrial Engineering Chemical Research 2001, 40, 4121-4140.

[5] R.Gundala,, K.A.Hoo, Multiple Model Adaptive control Design for a MIMOchemicalreactor,Industrial Engineering Chemical Research 38,1554-1563, 2000.

[6] D. Tyner, M. Soroush, Adaptive Temperature Control of Multi Product Jacketed, Reactor. .Industrial Engineering Chemical Research 38 4337-4344, 1999.

[7] I. Mcmanus, Adaptive Control; A QUT Avionics project, Queensland University of Technology; 2002.

[8] A. Rajagolan, G. Washegton; Simulink Tutorial, Ohio State University, 2002.

[9] M. Kozek, Mraclab-a Tool for Teaching Model Reference Adaptive Control; Vienna University of Technology, 2000. 